$\%$ Population single, widowed, or divorced 1981

Households with no car 1981

Average illegitimacy index 1982-1987

$\%$ Private households with no care 1981

$\mathrm{SPAR}_{\mathrm{asm}}-$ Standardised admission ratio based on district age, sex, and marital status composition

Standardised mortality ratio for ages under 65 , 1986

Standardised mortality ratio for ages under 75 , 1986

$\%$ Over 65s living alone, 1981

Standardised mortality ratio $1981-5$ bronchitis, emphysema and asthma

Average standardised mortality ratio 1981-7

Illegitimacy index 1986

$0 \cdot 503$

$0 \cdot 487$

$0 \cdot 481$

$0 \cdot 474$

$0 \cdot 471$

$0 \cdot 467$

3. REGRESSION EQUATIONS USED FOR DISTRICT MODELS

(standard error of coefficient shown in brackets)

Full model

SPAR $_{\text {asms }}=12+0.66 \times$ DRUG 1 STN $+0.82 \times$ SMR $($ IHD $)$ $( \pm 0 \cdot 10)$ $( \pm 0 \cdot 11)$

SPAR $_{\mathrm{asms}}=$ standardised psychiatry admission ratio $=$ actual admissions/age, sex, marital status expected admissions of residents, $1986 \times 100$
DRUGlsTN $=$ drug misusers, 1 st notifications $/ 10000$ resident population, 1988

SMR $($ IHD $)=$ standardised mortality ratio averaged $1981-5$ for ischaemic heart disease

Predicted admissions for each district are calculated as the predicted value of SPAR asms $_{\text {times the expected admissions }}$ based on the district's age, sex, and marital status structure and national psychiatric admission rates by age, sex, and marital status.

The model used explains $34 \%$ of the variation of SPAR values between districts. The predicted values of SPAR $_{\text {asms }}$ are calculated and multiplied by expected values to give predicted numbers of admissions.

The predicted admissions explain $79 \%$ of the variation between districts of actual admissions.

Simple model

Crude rate $=3.65+0.034 \times$ UPA SCORE

$$
( \pm 0 \cdot 0046)
$$

where:

Crude rate $=$ psychiatric admissions $/ 1000$ resident population UPA SCORE $=$ district underprivileged area score

Note that: (a) psychiatric admissions do not include mental handicap or psychogeriatrics; (b) figures apply to districts for 1986

\title{
Short term treatment of dermatophyte onychomycosis with terbinafine
}

\author{
M J D Goodfield, L Andrew, E G V Evans
}

Abstract

Objective-To evaluate the effect of short term treatment with terbinafine on dermatophytosis.

Design-Multicentre, randomised, double blind placebo controlled trial of $250 \mathrm{mg} /$ day terbinafine for 12 weeks in dermatophyte onychomycosis.

Setting-Eight dermatology centres in the United Kingdom.

Patients - 112 patients (mean age 44, range 19-78), 99 with mycologically proved toenail infections and 13 with fingernail infections, of whom eight were subsequently excluded and 19 failed to complete the study.

Intervention-Terbinafine $250 \mathrm{mg}$ daily or placebo for 12 weeks. Follow up for 36 weeks after stopping treatment.

Main outcome measures-Mycological cure (negative results on microscopy and culture) and clinical cure at the end of follow up, adverse events, and biochemical and haematological variables at monthly intervals during treatment.

Results-After follow up $82 \%(37 / 45)(95 \%$ confidence interval $68 \%$ to $92 \%$ ) mycological cure and $69 \%$ clinical cure were recorded for evaluable patients treated with terbinafine for toenail infection and $71 \%(5 / 7)(30 \%$ to $96 \%)$ mycological cure and clinical cure for those treated for fingernail infection. The corresponding values for those treated with placebo were $12 \%$ (3\% to $31 \%$ ) mycological cure and no clinical cure for toenail infections and $33 \%(1 \%$ to 91\%) mycological cure and no clinical cure for fingernail infections. On an intention to treat basis for toenail infections the figures were $73 \%(38 / 52)$ $(58 \%$ to $85 \%)$ mycological cure for terbinafine compared with $6 \%(0 \%$ to $30 \%)$ for placebo $(p<0 \cdot 007)$. Two withdrawals were related to adverse events with terbinafine, and there were no significant abnormal laboratory test results.

Conclusion-12 weeks' terbinafine is effective and safe treatment for nail dermatophytosis.

\section{Introduction}

Fungal infections of the nail (onychomycosis) are common and may occur in $2 \%$ to $5 \%$ of the population. Most involve dermatophytes (dermatophytosis), most commonly Trichophyton rubrum or Candida spp. Griseofulvin is the standard treatment for dermatophytosis of nails, being given orally for up to 18 months for toenail infections and for 12 months for fingernail infections. ${ }^{23}$ Results, however, are poor, and a cure is achieved in less than $40 \%$ of toenail and $70 \%$ of fingernail infections, ${ }^{4}$ with relapse of at least $20 \%$ at one year after apparently successful treatment. ${ }^{4}$ Thus an overall cure rate of less than $30 \%$ is achieved with this drug. Among newer antifungal agents, liver toxicity has limited the usefulness of oral ketoconazole to short term treatment,${ }^{5}$ itraconazole may be effective,${ }^{6}$ but is licensed for only four weeks' use, and topical application of tioconazole is probably of value only as adjunctive treatment ${ }^{\dagger}$; amorolfine remains unassessed in Britain.

Terbinafine is currently the most interesting of a new class of synthetic antimycotic agents, the allylamines. ${ }^{8}$ It may be taken orally and is fungicidal, inhibiting the fungal enzyme squalene epoxidase, which is important in the biosynthesis of ergosterol, an essential constituent of the fungal cell membrane. Inhibition of squalene epoxidase leads to a decrease in membrane ergosterol and accumulation of intracellular squalene, both of which are thought to contribute to the fungicidal action of terbinafine. ${ }^{9}$ This action is different from that of azole antifungals, which also inhibit ergosterol production in fungi, but by inhibiting lanosterol demethylase, an enzyme dependent on cytochrome $P-450 .{ }^{10}$ Other cytochrome $P-450$ linked enzymes are also inhibited by azole derivatives, though less with triazoles such as itraconazole. Possibly, these differences may explain the different side effects of these three groups of drugs. ${ }^{11}$

Terbinafine is effective in the treatment of dermatophyte infections of skin and nails, ${ }^{12-14}$ and its use 
for these conditions is now licensed in the United Kingdom in both hospital and general practice. Its effectiveness and safety in nail dermatophytosis was confirmed when given for up to 12 months for toenail infections and up to six months for fingernail infections. ${ }^{14}$ is The speed with which mycological cure was achieved and the known fungicidal action of the drug suggested that shorter courses of treatment might be successful for treating fungal nail disease. We report a multicentre study of the effectiveness of terbinafine given for three months for treating nail dermatophytosis.

\section{Patients and methods}

Patients with clinical subungual dermatophytosis were randomised (with random tables of Fisher and Yates) in a double blind, placebo controlled paralle group comparison of terbinafine $250 \mathrm{mg}$ nightly, given for 12 weeks and placebo. Sample size was calculated using a power of $90 \%$, with $\alpha$ equal to $5 \%$; a randomisation of 3:1 active to placebo treatment was chosen for ethical reasons, and this, assuming a mycological cure in $55 \%$ of patients taking the drug and in $20 \%$ taking placebo, required 81 subjects. To allow for $20 \%$ drop out it was intended that 100 patients would be recruited in eight centres in the United Kingdom.

Successive patients attending the dermatology outpatient departments of these centres who showed clinical evidence of nail infection were screened for inclusion in the study after written consent had been obtained. Patients with pre-existing renal, hepatic, or gastrointestinal disease and psoriasis or yeast infection of the nails and women who were pregnant or using inadequate contraception were excluded from the study. Mycological investigations were performed centrally in Leeds and comprised direct microscopy of nail clippings in $20 \% \mathrm{w} / \mathrm{v}$ potassium hydroxide, followed by culture on Sabouraud's glucose agar with added chloramphenicol $(0.05 \% \mathrm{w} / \mathrm{v})$ and actidione $(0.5 \% \mathrm{w} / \mathrm{v})$ at $27^{\circ} \mathrm{C}$ for up to three weeks. Only patients with a dermatophyte infection, confirmed by positive microscopy and culture before the start of treatment were eligible for inclusion in the trial. Patients with either fingernail or toenail infection were included and received identical treatment. After treatment, patients were followed up for a further 36 weeks, when a final evaluation of efficacy was made.

TABLE I-Reasons for failing to complete the trial in 27 non-evaluable patients

No of patients

Lost to follow up
Inadequate compliance
Negative mycological findings at baseline
Abnormal laboratory test results at baseline
Tonsillitis
Traumatic nail dystrophy
Breast carcinoma

9

TABLE II-Number (percentage) of mycological cures ${ }^{\star}$ in 85 patients with nail dermatophytosis who completed trial

\begin{tabular}{|c|c|c|c|c|}
\hline \multirow[b]{2}{*}{ Treatment } & \multicolumn{2}{|c|}{ Evaluation cohort } & \multicolumn{2}{|c|}{ Intention to treat } \\
\hline & Week 12 & Week 48 & Week 12 & Week 48 \\
\hline \multicolumn{5}{|c|}{ Toenail infection } \\
\hline $\begin{array}{l}\text { Terbinafine } \\
95 \% \text { Confidence interval (\%) } \\
\text { Placebo } \\
\quad 95 \% \text { Confidence interval (\%) }\end{array}$ & $\begin{array}{c}14 / 48+(29) \\
17 \text { to } 44 \\
3 / 25+(12) \\
3 \text { to } 31\end{array}$ & $\begin{array}{c}37 / 45(82) \\
68 \text { to } 92 \\
-\end{array}$ & $\begin{array}{c}18 / 60(30) \\
18 \text { to } 44 \\
4 / 28(14) \\
3 \text { to } 32\end{array}$ & $\begin{array}{l}38 / 52(73) \\
85 \text { to } 85 \\
1 / 16(6) \\
0 \text { to } 30\end{array}$ \\
\hline \multicolumn{5}{|c|}{$\begin{array}{cc}\text { Fingernail infection } \\
5 / 7(71) & 5 / 7(71) \\
30 \text { to } 96 & 30 \text { to } 96 \\
1 / 3(33) & - \\
1 \text { to } 91 & \end{array}$} \\
\hline
\end{tabular}

${ }^{\star}$ Negative results on microscopy and culture.

tData missing for one patient.
Patients who received placebo and failed to show improvement were offered active treatment after 16 or 24 weeks, depending on the views of the local hospital ethical committee. If they received treatment they were classified as placebo treatment failures.

Before starting treatment a full clinical examination and full biochemical and haematological investigations were performed and nail samples were examined mycologically. The number and site of infected nails were recorded, and the worst affected nail was chosen for specific measurement and observation. The proximal extent of nail involvement was marked by cutting a groove on the nail, and the distance from the proximal nail fold was measured. This measurement was repeated at each visit, allowing the appearance of unaffected nail to be recorded, as described by Zaias and Drackman. ${ }^{16}$

Patients were seen at monthly intervals throughout the treatment period. At each visit the mycological, biochemical, and haematological investigations were repeated; compliance and the occurrence of side effects were ascertained, and the target nail was examined clinically. Patients who experienced appreciable side effects or whose laboratory test results became abnormal were withdrawn at the discretion of the investigator.

The mycological cure rates at the end of the treatment period and of follow up were compared by the $\chi^{2}$ test. Mycological cure was defined as negative findings on microscopy and culture. The trial end points were thus 12 weeks (end of treatment) and 48 weeks (end of follow up).

\section{Results}

One hundred and twelve patients were enrolled into the study, 99 (70 taking terbinafine, 29 placebo) with toenail infection and 13 (eight taking terbinafine and five placebo) with fingernail infection. Seventeen patients had toe and fingernail involvement and were included in the toenail group because these infections are more difficult to treat. There were eight delayed exclusions because of negative mycological findings at baseline (five patients with negative cultures; three with negative results on microscopy), and 19 other patients failed to complete the study or were excluded from the analysis owing to significant violations of the study protocol (table I). In all, 85 patients ( 55 male, 30 female; mean age 44 , range 19-78) were suitable for evaluating efficacy of the drug: 75 had toenail infection, 49 of whom received terbinafine and 26 placebo, and 10 had fingernail infection, seven of whom received terbinafine and three placebo. Most $(80,94 \%)$ of these patients were infected with $T$ rubrum and the remainder with $T$ mentagrophytes.

Table II gives the mycological cure rates wth $95 \%$ confidence intervals. The cure rate at the end of treatment for patients with toenail infection treated with terbinafine was $29 \%$ (14/48; table II) and with placebo $12 \%(3 / 25)$. The number of patients with fingernail infection was small, but $71 \%(5 / 7)$ of those treated with terbinafine were mycologically cured at the end of treatment compared with $33 \%(1 / 3)$ in the placebo group. At the end of follow up $82 \%(37 / 45)$ of evaluable patients with toenail infection and $71 \%(5 / 7)$ of those with fingernail infection who had received terbinafine were mycologically cured. On an intention to treat basis the cure rates for toenail infection at the end of follow up were $73 \%(38 / 52)$ with terbinafine compared with $6 \%(1 / 16)$ with placebo. These differences are significant $(p<0.007)$ in favour of terbinafine $\left(\chi^{2}\right.$ test, two tailed). Acceptable clinical cure was defined as full, unaffected, normal nail growth. The clinical cure rates in the patients treated in the terbinafine were similar: $69 \%$ for toenail infection and 
$71 \%$ for fingernail infection at the end of follow up. No patient receiving placebo achieved an acceptable clinical response.

One measure of successful treatment is the rate of appearance of normal unaffected nail. ${ }^{16}$ The figure shows the rate of appearance of healthy nail in patients with toenail infection. For those taking terbinafine it was about $1 \mathrm{~mm} /$ month from the outset of treatment, indicating early control of infection, and was significantly different from that of the placebo group at 12 weeks (Mann-Whitney U test, $\mathrm{p}<0.001$ ) and at all points thereafter.

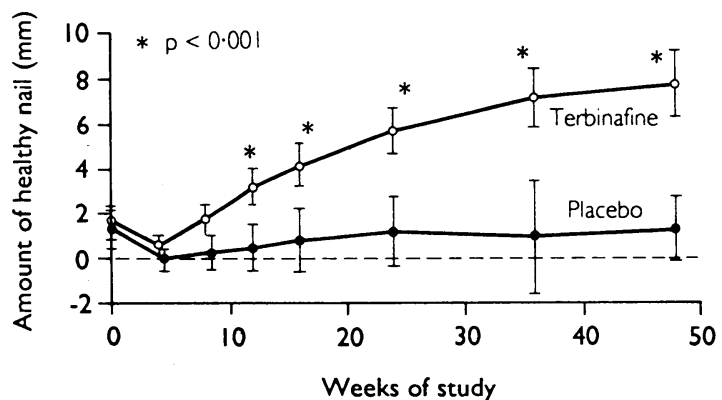

Change from baseline in unaffected nail length during treatment and follow up in patients with toenail infection. Bars are $95 \%$ confidence intervals

Of the failures classified as treatment failures on the basis of the patients' overall response, 13 were primary failures in that mycological cure was never achieved at any stage whereas in four the infection was considered to have relapsed as negative mycological results were achieved.

Table III shows recorded adverse events. Twenty six $(33 \%)$ patients treated with terbinafine reported adverse events compared with $14(41 \%)$ patients receiving placebo; during follow up a further 10 and 14 patients respectively reported adverse events. Two patients taking terbinafine stopped treatment, one (who was also taking antibiotics) with diarrhoea and one with a sore throat due to a bacterial infection. A patient who developed breast carcinoma (table I) was taking placebo. There were no important changes in laboratory variables.

TABLE III-Adverse effects in 85 patients receiving terbinafine or placebo by system

\begin{tabular}{lcc}
\hline System & $\begin{array}{c}\text { Terbinafine } \\
(\mathbf{n}=56)\end{array}$ & $\begin{array}{c}\text { Placebo } \\
(\mathbf{n}=29)\end{array}$ \\
\hline General & 6 & 3 \\
Central nervous system & 3 & 3 \\
Gastrointestinal & 18 & 12 \\
Ears & 1 & 0 \\
Eyes & 0 & 1 \\
Mouth & 4 & 0 \\
Musculoskeletal & 1 & 0 \\
Cardiovascular system & 1 & 0 \\
Psychiatric & 1 & 0 \\
Gynaecological & 2 & 0 \\
Respiratory & 5 & 0 \\
Skin & 9 & 3 \\
\hline Total & $51^{\star}$ & $22 \dagger$ \\
\hline
\end{tabular}

$\star 37$ Events reported during treatment, 14 during follow up.

†20 Events reported during treatment, two during follow up.

\section{Discussion}

Dermatophyte nail infection is not a serious problem in terms of overall morbidity and mortality, but it is a source of inconvenience, pain, and discomfort to individual patients, many of whom also find it cosmetically unacceptable. The infection contributes to and perpetuates the reservoir of dermatophyte fungi responsible for the spread of infection among those who use communal bathing facilities such as swimming pools and showers. Until now no really effective and safe treatment for dermatophytosis of nails has existed, though results obtained with long term terbinafine treatment are encouraging and suggest that this drug may be a major advance. ${ }^{15}$

Our study reinforces that view and indicates that long term treatment is not necessary for even the most severe and chronic nail infections. The cure rates achieved with three months' treatment were comparable to those found with 12 months' treatment - thus there seems to be no advantage in prolonging treatment beyond three months. Delayed clinical cure was noted in an earlier study ${ }^{17}$ and was seen again here. The clinical cure rates are almost certainly still underestimated because of the slow rate of growth of toenails and can be expected to increase further with time.

The drug was well tolerated, and shorter duration treatment is as safe as a course of six to 12 months. In this study the profile of adverse events was similar with terbinafine and placebo and, as in earlier studies, indicated that mild gastrointestinal upset is the major side effect. Encouragingly, there were no important changes in laboratory variables. Though unusual side effects may appear once terbinafine is widely used, the existing pharmacological and clinical evidence suggests that this is unlikely.

The patients in whom terbinafine treatment failed comprised two groups. The first included those who never achieved a mycological cure at any stage in the study, in whom the failures may be true failures, though late cure may still occur. The second included those who achieved a mycological cure but whose infection subsequently relapsed. With a short treatment course these relapses are probably true relapses rather than reinfections.

Terbinafine, the first orally active drug of the allylamine class of antifungal agents, is an effective and safe treatment for dermatophyte onychomycosis, and short term treatment is as effective as longer courses. This is a considerable advance in the management of dermatophyte nail infections. Of other available drugs, a comparison with itraconazole would be valuable, but given that itraconazole is a fungistatic drug against dermatophytes, terbinafine is likely to be more effective. Griseofulvin has remained the standard treatment for dermatophytosis but is relatively ineffective. Its main benefit is that it is cheap. However, three months of terbinafine treatment $(£ 137.40)$ costs little more than 18 months of griseofulvin treatment (£95.00) and cures about twice as many patients. The success of short term treatment makes the inevitably more expensive newer drug a viable option. Given this safety and efficacy profile, terbinafine should replace griseofulvin as the standard oral treatment for dermatophyte nail infections, even in primary care.

We thank our colleagues who allowed us to report their data: Drs Roger Allen and. Joe Sowden (Nottingham), Andrew Finlay and Lawrence Lever (Cardiff), John Wilkinson (High Wycombe), Dai Roberts (Glasgow), Rod Dawber (Oxford), John White (Southampton), Keith Freeman (Cambridge Military Hospital, Aldershot), and Professor Neville Rowell (Leeds); Mr Dick Forster (Regional Mycology Laboratory, Leeds) for his mycological expertise Sandoz UK for supplying terbinafine and supporting the study; and Mike Foreman and his staff at Data Analysis and Research, Kersewell, Lanarkshire, who organised, administered, supervised, and analysed the study.

\footnotetext{
1 Evans EGV. Nail dermatophytosis: the nature and scale of the problem. Journal of Dermatological Treatment 1990;1(suppl 2):47-8.

2 Rournal of Dermatological Treatment $199, \mathbf{1}$ (suppl 2$): 47-8$. Medical Publishing, 1990

3 Blank H, Roth FJ Jr. The treatment of dermatomycoses with orally administered griseofulvin. Arch Dermatol 1959;79:259-66.

4 Davies RR, Everall JD, Hamilton E. Mycological and clinical evaluation of griseofulvin for chronic onychomycosis. BMF 1967; iii:464-8

Heiberg JK, Sveigaard E. Toxic hepatitis during ketoconazole treatment. $B M \mathcal{F} 1981 ; 283: 825-6$.

6 Hay RJ, Clayton YM, Moore MK, Midgley G. An evaluation of itraconazole in the management of onychomycosis. Br f Dermatol 1988;119:359-66.

7 Hay RJ, Clayton YM, Moore MK. A comparison of tioconazole $28 \%$ nail
} 
solution versus base as an adjunct to oral griseofulvin in patients with onychomycosis. Clin Exp Dermatol 1987;12:175-7.

8 Petranyi G, Ryder NS. Stutz A Allylamine derivatives: new class of syntheric antifungal agents inhibiting fungal squalene epoxidase. Science 1984,224: antifungal agents inhibiting fungal squalene ep

Stutz A. Synthesis and structure-activity correlations within allylamine antimycotics. Ann N Y Acad Sci 1988;544:46-62.

10 Van de Bossche H, Willemsens G, Cools W, Lauwers WFJ, Lejeune L. Biochemical effects of miconazole on fungi. Inhibition of ergosterol biosynthesis in Candida albicans. Chemical and Biological Interaction 1978;21:59-78.

11 Back DJ, Stevenson P, Tjia JF. Comparative effects of two antimycotic agents, ketoconazole and terbinafine on the metabolism of tolbutamide, ethinyloestradiol, cyclosporin and ethoxycoumarin by human liver microsomes in vitro. Brf Clin Pharmacol 1989;28: 166-70.

12 Savin R. Successful treatment of chronic tinea pedis (mocassin type) with terbinafine (Lamisil). Clin Exp Dermatol 1989;14:116-9.
13 Villars V, Jones TC. Present status of the efficacy and tolerability of terbinafine (Lamisil) used systemically in the treatment of dermatomycoses of skin and nails. Fournal of Dermatological Treatment 1990;1(suppl 2):33-9.

14 Goodfield MJD, Rowell NR, Forster RA, Evans EGV, Raven A. Treatmen of of dermatophyte infection of the finger and toenails with terbinafin (SF 86-327, Lamisil), an orally active fungicidal agent. Br f Dermato

15 Zaias N, Serrano L. The successful treatment of finger Trichophyton rubrum onychomycosis with oral terbinafine. Clin Exp Dermatol 1989;14:120-3.

16 Zaias N, Drackman DA. A method for the determination of drug effectiveness in onychomycosis. I Am Acad Dermatol 1983;9:912-9.

17 Harland CC, Goodfield MJD, Evans EGV. A follow up study of terbinafine (SF-327, Lamisil) in the treatment of onychomycosis. Br $\mathrm{F}$ Dermato 1990;123(suppl 37):31.

(Accepted 24 February 1992)

\title{
Delayed diagnosis in non-insulin dependent diabetes mellitus
}

\author{
B M Singh, D M A Jackson, R Wills, J Davies, \\ P H Wise
}

\section{Department of \\ Endocrinology, Charing \\ Cross Hospital, London W6 8RF \\ B M Singh, senior registra \\ D M A Jackson, registrar \\ $\mathrm{R}$ Wills, sister, \\ diabetes care \\ J Davies, clinical \\ nurse specialist, diabetes \\ $\mathrm{P} \mathrm{H}$ Wise, consultant \\ endocrinologist}

Correspondence to: Dr Singh.

BMF 1992;304:1154-5
Disease duration is a recognised factor in the development of microvascular complications in non-insulin dependent diabetes, ' and delayed presentation might contribute to the prevalence of such complications at diagnosis. ${ }^{23}$ This study aims to examine the potential for achieving earlier diagnosis of non-insulin dependent diabetes.

\section{Methods and results}

We studied 100 newly diagnosed patients ${ }^{4}(36$ men, 54 women; mean age 60 (SD 11) years) presenting consecutively to the diabetes day centre at Charing Cross Hospital. The diagnosis had been made by general practitioners in 54 patients, by hospital departments in 33, during independent medical examinations in eight, and by the patients themselves in five. Patients were interviewed by standardised questionnaire to determine the nature and duration of their symptoms at presentation. Symptoms evaluated were thirst, polyuria, lethargy, and weight loss - the symptoms of diabetes most commonly known to nondiabetic people. ${ }^{5}$ The nature of contacts with doctors after symptoms had been recognised was recorded. Each patient marked the presence and duration of symptoms listed on a standard form to document those they had not previously volunteered.

A major symptom of diabetes had been reported to referring doctors by 39 patients. Nine patients had other specific or atypical presentations: four with infection (balanitis (2); abscess; necrotising fasciitis); four with visual disturbance (central retinal artery or vein occlusion (3); diabetic retinopathy); one with diabetic amyotrophy. The diagnosis was made in 31 patients presenting with symptoms not related to diabetes, and 21 were found at routine screening. At their first visit to the diabetes day centre 57 patients reported one or more of the major symptoms: 24 reported one symptom; 18, two; 14, three; and one reported having all four. Forty three patients still claimed to be asymptomatic (table). No individual symptom was widely volunteered, and thirst and polyuria in combination were recognised by only 17 patients. After completing the symptom inquiry form, 80 patients recognised the presence of one or more further symptoms: 35 , one; 26 , two; 17 , three; and 2 recognised four. Thirteen patients identified no further symptoms, but only seven continued to be truly asymptomatic (table). Taking reports of previously recognised and unrecognised symptoms together, all four symptoms were commonly reported (table).
Eighteen patients now recognised one major symptom; 14, two; 40, three; and 21, four. Fifty eight patients recognised thirst and polyuria in combination, with only 19 denying either. Overall, 39 patients had had symptoms for more than 12 months.

Numbers of newly presenting patients with non-insulin dependent diabetes $(n=100)$ categorised according to duration of previously recognised or unrecognised symptoms

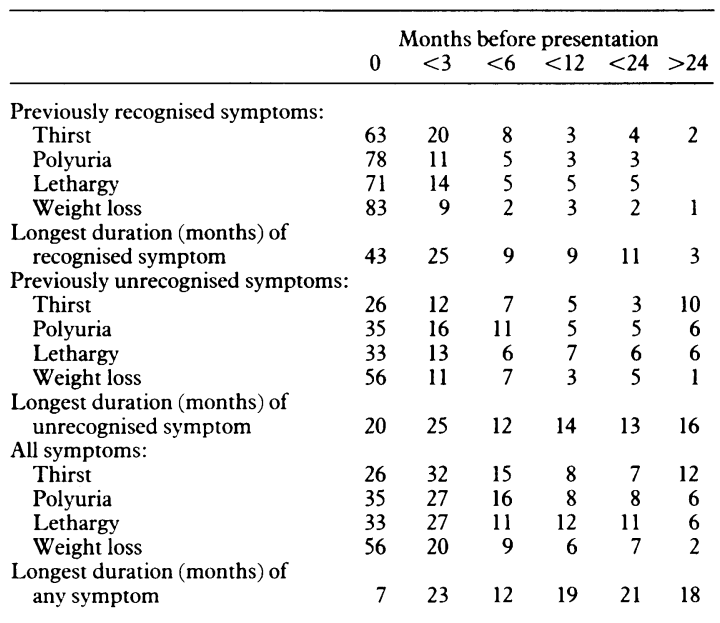

Seventy seven patients had seen a doctor while they had the recognised symptoms of diabetes. In 42 there had been no reason to suspect a diagnosis of diabetes. In eight patients the diagnosis had been made a median of 13 (range 3-60) months previously, with findings of raised random blood glucose'concentration in eight and glycosuria in seven, but the patients had not been informed. Seven of these patients had been seen in hospital departments. In 21 patients the diagnosis had been missed 6 (1-120) months before presentation. Of these, 12 had attended their general practice ( 3 with recurrent vaginal candidiasis, 1 with recurrent cellulitis, 8 with osmotic symptoms) and nine attended hospital departments ( 4 in ophthalmology ( 3 cataracts, 1 glaucoma), 2 in surgery ( 1 abscess, 1 "prostatism"), 2 in casualty (diabetic amyotrophy, recurrent abscess) and 1 in psychiatry). Urine had been tested in only one of these patients. Four patients had been informed of the diagnosis but failed to have further follow up for periods of 6-12 months before presentation. Thus, among 33 patients $(43 \%$ of those who had seen a doctor) the potential for treatment had existed 9 (1-120) months previously and occurred at hospital departments in 16 patients, in general practice in 13 , and after independent medical examinations in four.

\section{Comment}

Our data show that asymptomatic non-insulin dependent diabetes is unusual. Patients often have symptoms but do not recognise them as being abnormal. Almost 